

\title{
INVISCID DYNAMICS OF TWO-DIMENSIONAL SHEAR LAYERS
}

\author{
Kuei-Yuan Chien," Ralph E. Ferguson† \\ Naval Surface Warfare Center, Silver Spring, MD \\ Allen L. Kuhl** \\ Lawrence Livermore National Laboratory, Los Angeles, CA \\ Harland M. Glaz \\ University of Maryland, College Park, MD \\ Philip Colella \\ University of California, Berkeley, CA
}

\begin{abstract}
The dynamics of unconfined, spatially developing shear layers is studied by numerical solutions of the time-dependent Euler equations using a second-order Godunov scheme. Effects of density and velocity variations between the two streams of the shear layer are studied and color graphies is used to show more clearly the entrainment process of the surrounding streams. The calculations demonstrated that the evolution of the mean flow was dominated by two-dimensional, inviscid effects. The r.m.s. fluctuating velocity and density profiles were found to be in good agreement with the measurements of Oster and Wygnanski and of Konrad, except for the peak value of the $v^{\prime}$ profile.
\end{abstract}

\section{INTRODUCTION}

This paper presents results of numerical simulation of unconfined, spatially developing, two-dimensional unsteady shear layers in a constant-ambient-pressure environment. It has been known for more than a hundred years that the shear layer generated by a splitter plate can be inviscidly unstable. The late-time consequence of such flow instabilities is that perturbed shear layers roll up into large-scale vortical structures (Michel 1932; Brown and Roshko 1974); the latterwork also demonstrated that the largest-scale structures are

\footnotetext{
- Senior Aerospace Engineer, Associate Fellow AlAA

tMathematician

- Senior Scientist

\$Associate Professor, Mathematics Department, Associate Fellow AIA A

\$Associate Professor, Mechanical Engineering

Department, Member AlAA
}

essentially independent of the flow Reynolds number (although micro-scale mixing within these structures is Reynolds-number dependent). It also is known that the growth of the shear layer is governed by the pairing mechanism of these vortical structures (Winant and Browand 1974); a complex nonlinear dynamical process (Corcos and Sherman 1984). Perturbations may occur naturally or they may be intentionally applied. Studies with applied upstream perturbations indicate that vortex dynamics are basically inviscid (Ho and Huang 1982; Oster and Wygnanski 1982).

Many shear layer computations involving discretizations of the Navier-Stokes equations are available. These include results using finite-difference methods (Corcos and Sherman 1984; McInville et al. 1985; Davis and Moore 1985), spectral methods (Riley and Metcalfe 1980), and vortex methods (Ashurst 1979; Leonard 1980; Ghoniem et al. 1982). All of these calculations succeed in resolving small-scale flowfield structures and are of high quality. However, modeling of the viscous terms imposes computational inefficiencies due to the disparity between viscous and inviscid length scales. One may either resolve the viscous length scales on a mesh and, hence, use severely restricted time steps, or employ implicit time differencing and accept the resulting numerical diffusion. The random vortex method (Chorin 1973, 1986) avoids this problem at the expense of introducing a statistical error.

Due to advances in the development of high-order upwind methods for solving conservation laws, it is now possible to compute high resolution, essentially nondiffusive flowfields for the equations of inviscid gas dynamics. This approach is taken here to simulate shear layer experiments, with the objective of demonstrating that viscous forces need not be taken into account in modeling the dynamics of shear layer 
flowfields. Such methods neglect molecular diffusion effects (i.e., diffusion of vorticity and passive scalars across streamlines), so that the methodology may be viewed as an asymptotically large Reynolds number approach. An advantage of using the gas dynamic equations is that baroclinic generation of vorticity is automatically included. Its main disadvantage is that small Mach number calculations can be quite expensive; this will be explained in more detail in Section 2.

3

Previous calculations along these lines have been reported by Grinstein et al. (1986), using flux-corrected transport code to solve Euler's equations, and by Inoue (1985), using a vortex method to solve potential equations. Obviously, steady upstream conditions will not produce any vortex formation using an Euler code. A fluctuating inflow-pressure condition was used by Grinstein et al. (1986) by imposing a zeroslope condition on the pressure at the inflow boundary. Therefore, the pressure perturbations are not prescribed; in fact, the quantitative values of these perturbations are part of the solution. We chose to follow our previous work (Chien et al. 1987) by imposing a given velocity perturbation at the inflow (upstream) boundary, with the frequency content and relative amplitudes of the perturbations obtained from the inviscid linear spatial instability calculations of Monkewitz and Huerre (1982). (More details are given in Section 2.) In addition, quantitative r.m.s.fluctuating velocity results, which require long computations to obtain converged solutions, are not available in Grinstein et al. (1986). In fact, despite the large amount of computational efforts, detailed quantitative mean and fluctuating flow calculations are quite meager; all of those reported in the literature have been based on vortex methods (Ashurst 1979; Inoue 1985; Inoue and Leonard 1987; Ghoniem and $\mathrm{Ng} 1987$ ), which cannot yet be generalized to study the variable density effects investigated by Brown and Roshko (1974) and Konrad (1977). Therefore, the primary purpose of this paper is to present detailed quantitative results based on a finite-difference solution of the Euler's equations and to compare these solutions with available experiments and other pertinent computations. Effects of both the density ratio and velocity ratio on the solutions are addressed in detail in the paper.

The numerical results presented here were obtained using an explicit second-order Godunov scheme (Colella and Glaz 1985). The nondiffusive, high resolution properties of this scheme have been carefully demonstrated by a mesh refinement study combined with a comprehensive comparison with other schemes (Woodward and Colella 1984). Its accuracy has been shown in a variety of applications (Colella et al. 1985, Glaz et al. 1985); in many situations, embedded shear layers become unstable, roll up, and interact with the ambient flow as well as self-interact (Glowacki et al. 1986, Kuhl et al. 1987). Thus, the present calculations also serve to validate the accuracy of the code in shear layer regions, by direct qualitative and quantitative comparison with experimental data.

By design, our work is limited to unsteady calculations of two-dimensional shear layers which are statistically steady (in the sense of time-averaging); as suggested by Roshko (1981), a two-dimensional solution is valuable both as a gauge against which to compare the real flows, and as a step in understanding them. Extension of the present method to three-dimensional cat culations using operator splitting is conceptually feasible, but the computational resources required for such an effort is extremely prohibitive at the present time. Some of the results herein have been presented elsewhere in preliminary versions of this work (Chien et al. 1988, 1989; Kuhl et al. 1990). In these earlier reports, monitoring stations situated at three constant streamwise locations were used to store the flowfield time histories for quantitative data analysis. Since the upstream condition is known to have a strong effect on the development of a free shear layer (Bradshaw 1966), more monitoring stations are needed to study the rate of attaining self similarity of various mean and fluctuating profiles. Nineteen monitoring stations located at constant streamwise intervals throughout the region of interest have been used in three new calculations to be presented in this study. Two of these calculations correspond to the cases studied experimentally in detail by Brown and Roshko (1974) and by Oster and Wygnanski (1982), respectively. The density ratio and the velocity ratio for these two cases are both different. Since the development of a free shear layer is known to depend on both of these ratios, a third calculation bas also been made so that the effect of each parameter can be individually assessed.

The formulation of our numerical simulations is described in Section 2. Results and comparison with experimental data are presented in Section 3, where detailed quantitative comparisons of mean and fluctuating flowfield profiles are offered (the numerical data are obtained by statistically averaging the numerical solution over time intervals which are long relative to the time scales of vortex formation and pairing). Our conclusions are summarized in Section 4. 


\section{Methodology}

Our computational models are based on the equations of inviscid gas dynamics, thereby neglecting molecular viscosity and heat conduction; gravitational forces are neglected as well. The equations may be written in conservation form for a Cartesian geometry as

$$
\mathbf{U}_{1}+\mathbf{F}(\mathbf{U})_{x}+\mathbf{G}(\mathbf{U})_{y}=0
$$

where

$\mathbf{U}=\left(\begin{array}{c}\rho \\ \rho u \\ \rho v \\ \rho \mathrm{E}\end{array}\right), \mathbf{F}=\left(\begin{array}{c}\rho u \\ \rho u^{2}+p \\ \rho u v \\ \rho u \mathrm{E}+u p\end{array}\right), \mathbf{G}=\left(\begin{array}{c}\rho u \\ \rho u v \\ \rho v^{2}+p \\ \rho v \mathrm{E}+v p\end{array}\right)$,

and $\rho$ is the density, $u=(u, v)$ is the velocity field in the Cartesian coordinates $x=(x, y), p$ is the pressure, $\mathbf{E}=e+\frac{1}{2}\left(u^{2}+v^{2}\right)$ is the total specific energy, and $e$ is the specific internal energy. We always assume a perfect gas with equation-of-state

$$
p=(\gamma-1) p e,
$$

and take $\gamma=1.4$. The streamwise direction is always taken to be the positive $x$-axis.

The Eulerian second-order Godunov scheme (Colella and Glaz 1985) is used to solve these equations for given initial data and boundary conditions. This scheme is in conservation form and explicit.

Dimensional operator splitting is used for two-dimensional calculations. A rectangular $\mathbf{N} \times \mathbf{M}$ mesh is specified by defining zone interfaces $x_{i}, i=0, \ldots$, $\mathrm{N}$ and $y_{j}, j=0, \ldots, \mathrm{M}$ with zone sizes $\Delta x_{i}$ and $\Delta y_{j}$ where $\Delta x_{i}=x_{i}-x_{i-1}$ and $\Delta y_{j}=y_{j}-y_{j-1}$. Flowfield quantities, averaged over a computational zone, are denoted by $\rho_{i j}$, etc. The time step is limited by the CFL stability criterion

$$
\Delta t=\sigma \min _{i, j}\left(\frac{\Delta x_{i}}{\left(c_{i j}+\left|u_{i j}\right|\right)}, \frac{\Delta y_{j}}{\left(c_{i j}+\left|v_{i j}\right|\right)}\right)
$$

where $c=(\gamma p / \rho)^{1 / 2}$ is the sound speed and $\sigma<1$ is the Courant number. The value $\sigma=0.95$ has been used in all calculations.

Such explicit methods are most appropriate for compressible flowfields. Small Mach number flowfields may also be obtained, but at the cost of either very poor computational efficiency or violation of Mach number similitude. For example, if the Mach number $M \sim 0.01$ (which is typical of some of the experimental data used here), then it would take approximately one hundred time steps to convect a fluid parcel through one computational zone. To avoid this problem, the sound speed of the gas has been modified for the computations so that typical flow Mach numbers would be of the order $M \sim 0.2$. Of course, the density and velocity ratios across the shear layer are preserved, but Mach number similitude is violated. Since compressibility effects are negligible at these Mach numbers and we do not require temperature data for our comparisons, this Mach number compromise was found acceptable for the present study.

The dynamics of the interface separating the two fluid streams is of great importance in shear layer studies. Consequently, an additional calculation was performed of a discretization of the material interface by marker particles, $x_{k}(t), k=1, \ldots, K(t)$ where $x_{k}(t)$ denotes the location of the $k$ th marker at time $t$ and $K(t)$ is the total number of markers present in the flowfield at time $t$. The time evolution of the markers was governed by passive advection, i.e., $d \mathbf{x}_{k} / d t=u\left(x_{k}, t\right)$; this ordinary differential equation with right-hand side given by the underlying computed velocity field was solved by straightforward finite differences and linear interpolation for $u$. Initially, the interface is represented by several thousand markers and new markers are inserted at the inflow during every time step. Since the interface is unstable, it is expected that $\left|x_{k}-x_{k-1}\right|$ can become large; whenever this value exceeded twice the initial marker spacing, a new marker was inserted at their midpoint. Marker points which exited the finegrid region (see below) were deleted. Finally, the condition $K(t) \leq 75,000$ was imposed due to computer time limitations; if the condition was violated, no further markers would be inserted over the last forty percent of the mesh in the streamwise direction.

The accuracy of this approach is a matter of some debate. Due to the underlying physical instability, one is solving what amounts to a chaotic dynamical system in current terminology. In particular, expo nential separation of orbits is expected which implies that the numerical orbit $x_{k}(t)$ is not close to the actual physical orbit of the initial marker after a time which is probably small relative to the total computation time. However, this does not imply that the aggregate of numerical orbits is not a good representation of the aggregate of physical orbits. Indeed, our results here (comparisons with contour plots and shadowgraphs from the experiments) suggest the contrary.

The calculations were performed on a two dimensional Cartesian grid, see Figure 1. A fine, uniformly-zoned 
region with $\Delta x=\Delta y$ covering the domain $D_{F}=$ $\left\{(x, y): 0 \leq x \leq X_{\mathrm{F}},-Y_{\mathrm{F}} \leq y \leq Y_{\mathrm{F}}\right\}$ was placed straddling the centerline of the shear layer. An expanding grid (above, below, and to the right of $D_{F}$ ) was used to place the mesh boundaries far from the region of interest and thereby minimize any influence of the numerical boundary condition on the dynamics of the free shear layer. For this study, we have taken $\Omega_{\mathrm{F}}=500 \times 80$ and $\Omega_{\mathrm{T}}=520 \times 114$, where $\Omega_{\mathrm{F}}$ and $\Omega_{\mathrm{T}}$ refer to the grids in the fine and total mesh regions, respectively.

Initial data was determined using a $\operatorname{Tanh}(y)$ profile to model the shear layer. We have

$$
\begin{aligned}
& u(x, y, t=0)=f(y) \\
& v(x, y, t=0)=0
\end{aligned}
$$

where

$$
f(y)=U_{m}[1+\lambda \operatorname{Tanh}(2 y / \delta)]
$$

and

$$
U_{m}=\frac{\left(U_{1}+U_{2}\right)}{2}, \quad \lambda=\frac{\left(U_{1}-U_{2}\right)}{\left(U_{1}+U_{2}\right)} .
$$

Here, $U_{1}$ and $U_{2}$ denote the free stream velocity at $y= \pm \infty$, respectively; $U_{m}$ is the mean flow velocity; $\lambda$ is the shear parameter and $\delta$ is the maximum slope thickness of the layer, i.e., $\delta=\left(U_{1}-U_{2}\right) /(d u / d y)_{\max }$. If the free stream densities are different, we have

$$
\rho(x, y, t=0)=h(y)
$$

where

$$
\begin{aligned}
h(y) & =\rho_{m}\left[1+\lambda_{\rho} \operatorname{Tanh}(2 y / \delta)\right] \\
\rho_{m} & =\frac{\left(\rho_{1}+\rho_{2}\right)}{2}, \quad \lambda_{p}=\frac{\left(\rho_{1}-\rho_{2}\right)}{\left(\rho_{1}+\rho_{2}\right)},
\end{aligned}
$$

where $\rho_{1}, \rho_{2}$ are the free stream densities at $y= \pm \infty$, respectively; $\rho_{m}$ is the mean shear layer density and $\lambda_{p}$ is the density shear parameter. Note that the same $\delta$ is used as the length scale for the density distribution.

The left inflow boundary data are determined from

$$
\begin{aligned}
& u(0, y, t)=f(y) \cdot g(t) \\
& v(0, y, t)=0 \\
& \rho(0, y, t)=h(y)
\end{aligned}
$$

where

$$
g(t)=1+\sum_{n=1}^{N} \epsilon_{n} \sin \left(\omega_{n} t\right)
$$

The perturbation frequencies $\omega_{n}$ and amplitudes $\epsilon_{n}$ have been taken from the lineat stability analysis for
$\operatorname{Tanh}(y)$ shear layer profiles (Monkewitz and Huerre 1982):

$$
\begin{gathered}
\omega_{1}=0.438 ; \omega_{n}=\omega_{1} / n, n=1, \ldots, N, \\
\epsilon_{1}=0.01, \epsilon_{2}=0.75 \epsilon_{1}, \epsilon_{3}=0.55 \epsilon_{1}, \epsilon_{4}=0.44 \epsilon_{1}, \\
\epsilon_{n}=1.7 \epsilon_{1} / n \text { for } n \geq 5 .
\end{gathered}
$$

Note that the maximum perturbation amplitude is one percent and $\omega_{1}$ is twice that given by Monkewitz and Huerre (1982) because our reference length scale is twice of theirs (see below).

Numerical implementation of the boundary conditions is straightforward (Colella and Glaz 1985) for free shear layers. Dirichlet data is imposed on the upstream side (using the equations above) as well as the top and bottom sides (using free stream values) and an outflow boundary condition is used on the downstream side.

The lengths have been normalized by $\delta / 2$ and we choose $\Delta x=\Delta y=1$ in the fine zone region; i.e., $X_{F}=500$ and $Y_{F}=80$. Also, $X_{R}=8800$ and $Y_{T}=1500$ (see Fig. 1 for definitions of $X_{F}, X_{R}$, $Y_{F}$ and $Y_{T}$ ); it may be noted how rapidly the grid expands from the fine mesh 10 cover the total computational domain. Despite the excellent agreement with experiment, it would be of great interest to perform a mesh refinement study and re-analyze detailed flow-field structures. With the code used here, this is currently beyond the limits of our computer resources.

The physical parameters defining the simulations are summarized in Table 1. The parameter $r$ is defined by $r=U_{2} / U_{1}$ and $M_{1}, M_{2}$, and $M_{m}$ refer to the Mach numbers of the top stream, bottom stream, and the mean flow, respectively. In all of the calculations, $U_{m}=1.0$ (i.e., the velocities are normalized by $U_{m}$ ), the density is normalized by the lower ambient density, and we take $p=p_{1}=p_{2}=17.86$; if $\rho=1$, then this choice implies that the sound speed $c=5.0$ and $M_{m}=0.2$.

Table 1. Calculational Matrix

\begin{tabular}{|r|c|c|c|c|c|c|c|c|c|c|}
\hline Case & $U_{1}$ & $U_{2}$ & 1 & $\lambda$ & $n$ & $\lambda_{1}$ & $\lambda_{1}$ & $M_{1}$ & $M_{2}$ &.$I_{0}$ \\
\hline 1 & 1.45 & 0.55 & 0.38 & 0.45 & 1.0 & 7.0 & -0.75 & 0.29 & 0.29 & 0.40 \\
$I 1$ & 1.45 & 0.55 & 0.38 & 0.45 & 7.0 & 1.0 & 0.75 & 0.77 & 0.11 & 0.40 \\
III & 1.25 & 0.75 & 0.60 & 0.25 & 1.0 & 1.0 & 0 & 0.25 & 0.15 & 0.20 \\
IV & 1.45 & 0.55 & 0.38 & 0.45 & 1.0 & 1.0 & 0 & 0.29 & 0.11 & 0.20 \\
\hline
\end{tabular}




\section{RESULTS AND DISCUSSIONS}

A typical shear layer contains a wide spectrum of perturbation frequencies, and the mixing width of the layer continues to grow with increasing distance. The numerical simulation of one such experiment (Brown and Roshko 1974) is described here in detail. Additionally, the calculations were continued so as to provide data for a statistical analysis of mean and fluctuating fowfield quantities of the solution, and these are compared with experimental data. This analysis is repeated for three other simulations - one using the same initial conditions as in the above experiment but with the density ratio inverted (Brown and Roshko 1974), and two more referring to an experiment (Oster and Wygnanski 1982) with equal density freestreams. The flow parameters for all four cases are listed in Table 1.

The calculation for Case I began by exciting only the fundamental frequency for 1000 time steps. At this point, the first nine subharmonics $\omega_{2}, \omega_{3}, \ldots, \omega_{10}$ were included in the inflow boundary perturbation and retained for the remainder of the calculation. All ten modes were used from the beginning for Cases III and IV. We computed a total of 20,000 time steps for Case 1, 30,000 steps for Case III and 26,000 steps for Case IV. The Case II flow.field was initialized using the results of the Case I computation at time $t=2579.0$ at which point the $\operatorname{Tanh}(y)$ profile for the density was inverted and the new calculation commenced. A total of 14,000 time steps were computed for Case II (although $\sim 2000$ steps were required for the influence of the initialization procedure to exit the computational mesh). Each time step took about 4 seconds on the LANL CRAY XMP computer.

The dynamic evolution of the Case 1 flowfield was discussed in Kuhl et al. (1990) using material interface plots. For brevity, one such plot is shown in Figure 2(a). This material interface plot actually represents unconnected dots placed at the marker particle locations. The initial interface contained 5,000 equally spaced markers. This number rapidly increased to the imposed maximum of 75,000 during the course of the calculation, which implies that the length of the interface (insofar that this remains meaningful) increased by $a$ factor greater than 15 during the course of the calculation. We note that in the experiment, the upper gas was helium and the lower gas was nitrogen; this terminology is used below for convenience.

For comparison with this calculated interface, Figure 2(b) presents a shadowgraph picture of the helium-nitrogen interface recorded during the exper- iment (Brown and Roshko 1974, Fig. 3d). Similarities between the calculated and experimental interface are remarkable. The shape and wavelength of the largescale structures are quite similar.

Also included in Figure 2 are the corresponding density, vorticity and overpressure contours. The shape of the large structures as deduced from the density contours agrees with the material interface shape, indicating little numerical diffusion. It is interesting to note that vorticity is created near the braid regions and entrained into the structures. Approximately circular low pressure regions are found in each large structure, and this generates a radial pressure gradient. As the helium-nitrogen interface is entrained obliquely through this pressure gradient, vorticity is generated by the baroclinic mechanism. The maximum value of this vorticity reaches about minus one-half of the inflow vorticity; hence, it must have an effect on the flow. Indeed, close inspection of the material interface reveals some smaller-scale vortices rotating in the counterclockwise direction. This causes the merging patterns to be considerably more complex and less regular than the other cases to be discussed later.

Figure 3 depicts the corresponding flowfield near the centerline (i.e., $y=0.5$ ). Large fuctuations are evident in the velocity, density and dynamic pressure. In fact, densities corresponding to pure helium and to pure nitrogen are seen near the centerline; similarly, the velocity fluctuates between values corresponding to approximately $U_{1}$ and to $U_{2}$. Overpressure fluctuations are small (between +1.5 to -4.0 percent of ambient), which validates the use of the Mach number compromise $(0.29 \leq M \leq 0.4)$ for this calculation.

To gain some insight into the entrainment process, density and vorticity contours for Case $I$ are shown in color in Figure $\dot{4}$, where the light fluid is shown as blue and the heavy fluid as red. In agreement with Grinstein et al. (1986), the density color visualization show's clearly that more high-speed (light) fluid are entrained into the shear layer than low-speed fluid. The vorticity color visualization shows the formation and growth of the large-scale vortical structures somewhat more clearly than the contours shown in Figure 2(d). The baroclinic generation of vorticity (due to density and pressure gradients being nonparallel), shown as blue, and the vorticity due to velocity gradients, shown as red, are vividly seen.

Material interface plots of the first three cases are compared in Figure 5. One notes immediately that the large-scale structures of Case II are considerably less 
complex than those of Case 1. The visual agreement between these plots and the shadowgraphs of the experiments is remarkable. The large-scale dynamics of the equal-density Case III layer is much more straightfor ward in that relatively little small-scale entrainment and interleaving of the two streams is apparent in the vortical structures. In Figure 6 , the calculated visual spreading rates $\delta_{v i s}^{\prime}$ are compared with the experimental data (Brown and Roshko 1974, Fig. 7). The bars over the calculated results represent the variation in the visual spreading rate over time and the straight lines are suggested by Brown and Roshko (1974). The calculations are in good agreement with the experimental data. Other measures of the spreading rates based on the mean velocity profiles will be presented later.

In order to implement our statistical analysis, monitoring stations were used to store the flowfield time histories. Each station consists of 82 positions in the fine zone region of the mesh across the shear layer. Except for Case II, nineteen stations located at constant intervals between $x=25$ and $x=475$ were employed. These flowfield histories were then integrated over the last 10,000 time steps to establish the various timeaveraged shear layer profiles. For Case II, three stations located at $z=100,200$ and 400 were employed and only the last 6000 time steps were available at all 246 positions for the time integration. One reason for not doing an equally elaborate study for Case II is because the mixing is less vigorous than Case I and bence, less interesting. In addition, no experimental fluctuating data are available for Case II for comparison; 6000 time steps are adequate to establish mean profiles.

Several measures of the spreading rate of the shear layer based on these time-averaged mean velocity profiles have been calculated in the present study. Following previous investigators (Liepmann and Laufer 1947; Oster and Wygnanski 1982; Inoue and Leonard 1987), we introduce the notation $y_{p}$ to denote the location at which the mean streamwise velocity component $\bar{u}$ reaches the value given by

$$
\bar{u}=U_{2}+p\left(U_{1}-U_{2}\right)
$$

Of particular interest are the quantities $y_{0.5}$ and $b\left(\equiv y_{0.95}-y_{0.1}\right)$. Their values at all 19 stations have been computed for Cases I, III and IV; they exhibit good linear behavior over the last 15 stations. A leastsquares linear fit over these data allows us to compute the slope and the corresponding location of the virtual origin $x_{0}$. We have also calculated the momentum thickness (for a constant-density flow)

$$
\theta=\int_{-\infty}^{\infty} \frac{\left(U_{1}-\bar{u}\right)\left(\bar{u}-U_{2}\right)}{\left(U_{1}-U_{2}\right)^{2}} d y
$$

for the same cases and obtained the slope and the corresponding $x_{0}$ by the same procedure. The values of $\frac{d b}{d x}, \frac{d 0}{d x}$ and $\frac{d y e . s}{d x}$ are listed in Table 2 together with the experimental data of Oster and Wygnanski (1982) and the computed values of Inoue and Leonard (1987). Several conclusions may be drawn from Table 2.

\begin{tabular}{|c|c|c|c|c|c|}
\hline Source & $\lambda$ & $\lambda_{1}$ & $\frac{d b}{d s}$ & $\frac{d 0}{d x}$ & $\frac{d y e x}{d x}$ \\
\hline Oster and W'sgmanski & 0.25 & 0 & 0.045 & 0.009 & .0 .004 \\
\hline Inove and Leonard & 0.25 & $\mathbf{0}$ & 0.049 & 0.010 & .0 .006 \\
\hline This study & 0.25 & 0 & 0.050 & 0.010 & .0 .006 \\
\hline Oster and Wgnanski" & 0.45 & 0 & 0084 & 0.016 & .0 .018 \\
\hline This study & 0.45 & 0 & 0.081 & 0.016 & .0 .016 \\
\hline This study & 0.45 & -0.75 & 0.089 & 0.015 & 0.021 \\
\hline
\end{tabular}

Table 2. Comparison of Various Sprcading Rates

First of all, for constant-density cases, present finitedifference results agree well with the experimental data and the solution of a vortex method, indicating the reliability of the present approach. Secondly, the growth rate of the shear layer (as measured by either $\frac{d b}{d x}$ or $\left.\frac{d d}{d x}\right)$ and the slope of its center line $\left(\frac{d y_{0.3}}{d x}\right)$ increase in magnitude with increasing $\lambda$. As pointed out before by other investigators, the center line of the constantdensity sheas layer moves toward the low-speed region (Oster and Wygnanski 1982), indicating that more high-speed fluid particles are entrained into the shear layer than low-speed fluid particles (Grinstein et al. 1986). As the density of the high-speed fluid is re duced relative to the low-speed fluid (as e.g., in Case I), these lighter particles are easier to be entrained into the shear layer, resulting in a higher spreading rate than that of the corresponding constant-density case. This has already been shown in Figure 6 using visual spreading rate data, and the $\frac{d b}{d x}$ values given by the present calculations listed in Table 2 reconfirm this reasoning. One may note that the growth rate based on visual data (as in Fig. 6) is more sensitive to density variations than $\frac{d b}{d x}$. More surprising is the failure of $\frac{d \theta}{d x}$ to indicate even the trend of this density effect. For variable-density flows, the momentum thickness should be modified by

$$
\theta^{\prime}=\int_{-\infty}^{\infty} \frac{\rho\left(U_{1}-\bar{u}\right)\left(\bar{u}-U_{2}\right)}{\rho_{m}\left(U_{1}-U_{2}\right)^{2}} d y
$$


and our calculations give $\frac{d 0^{\prime}}{d x}=0.006$ for Case $1, \mathrm{a}$ value which is two and a half times smaller than the corresponding value for the constant-density Case IV. This may be explained as follows. As the density of the high- velocity fluid is reduced, the average velocity of this particle and a heavier low-velocity particle will be lower than the average velocity of these two particles at constant density. Therefore, the center line of this shear layer moves toward the high-velocity side relative to the corresponding constant-density flow. For Case $I, p_{2}=7 p_{1}$, and the density reduction on the highvelocity side is sufficient to yield $\frac{d y_{0} .}{d x}>0$. The region over which the product $\left(U_{1}-\tilde{u}\right)\left(\bar{u}-U_{2}\right)$ is the largest occurs now where $\frac{R}{p_{m}} \ll 1$, therefore, producing a smaller value of $\theta^{\prime}$ than the corresponding constantdensity case (i.e., $\theta$ ). Since $\theta=\theta^{\prime}=0.5$ at $x=0$, it is easy to see that $\frac{d \theta^{\prime}}{d x}<\frac{d \theta}{d x}$. Consequently, we conclude that the slope of the momentum thickness is not indicative of the extent of mixing in a variable-density shear layer.

To compare time-averaged profiles at different stations, a scaling variable

$$
\eta=y /\left(x-x_{0}\right)
$$

will be used, with the virtual origin, $x_{0}$, determined from the least-squares linear fit of $b$ for Cases I, III and IV. Since only three stations are available for Case II, a reliable curve fit cannot be made. Therefore, $x_{0}$ determined from material interface plots is used for that case. The results are: $x_{0}=13.5,-42.0,31.0$ and -2.0 for Cases I, II, III and IV, respectively.

The development of the mean velocity and density profiles for Case I is shown in Figure 7. It is clear that the profiles do approach an asymptotic, similar shape as $x$ is increased, and the above scaling helps to collapse the data. In fact, these figures indicate that the profiles at $\frac{x}{\theta_{1}}=300$ are not far from the asymptotic ones $\left(\theta_{i}\right.$ is the initial momentum thickness, $\left.\theta_{i}=0.5\right)$. The development of the mean velocity profiles for Cases III and IV is shown in Figures 8 and 9, respectively. It appears that, for these constant-density flows, the above scaling again helps to collapse the data; the mean profiles are somewhat less complicated and the profile at $\frac{x}{\theta_{1}}=200$ is not far from the asymptotic distribution.

The mean velocity and density profiles at selected stations for Cases I, II and III are compared with the mean profiles measured by Brown and Roshko (1974, Figs. $13 \mathrm{~b}$ and $c)$ and Oster and Wygnanski (1982, Fig. 6d) in Figures 10-12. The calculated profiles are seen to agree reasonably well with the measured profiles. The best agreement between measurements and calculations is for the simpler, constant-density flow (Case III).

We have also computed the r.m.s. time averages about the mean values. The development of the fluctuating r.m.s. quantities $u^{\prime}, v^{\prime}, \rho^{\prime}$ and shear stress $\left(\overline{-u^{\prime} v^{\prime}}\right)$ for Cases I, III and IV is shown in Figures 13-15. We start by considering the simplest, constant-density, low shear case shown in Figure 13 (Case III). The fluctuating velocity profiles are seen to evolve from low intensities to much higher, well- developed distributions, and the lengths for these fluctuating profiles to reach their asymptotic states are longer than that for the mean velocity profiles. Over the development region, $u^{\prime}$ profiles sometimes show the double-peaked shape similar to that occurring in the Region II of forced shear layer studied by Oster and Wygnanski (1982, Figs. 20 and 21 ), but $v^{\prime}$ profiles remain essentially bell-shaped. Note that the development of the shear stress is less orderly than either $u^{\prime}$ or $v^{\prime}$, with perhaps a longer developmental length. Note also that the fluctuating profiles are not as converged as the mean profiles shown in Figure 8.

The corresponding profiles for higher shear (Case IV) are shown in Figure 14. Comparing to the lower shear case, the fluctuating profiles extend to a wider lateral distance, as was observed in experiments (Oster and Wygnanski 1982), and, in agreement with stability theory (Monkewitz and Huerre 1982), the rate of amplification of the first few profiles increases with higher shear. The shear stress distributions converge at an even slower rate, but the overall trends of these two constant-density cases are qualitatively similar.

When the density of the two freestreams are not equal, the fluctuating profiles become more complicated. Shown in Figure 15 are the $u^{\prime}, v^{\prime}$, shear stress and $\rho^{\prime}$ distributions for Case 1. Comparing to the corresponding constant-density results shown in Figure 14, we see that $u^{\prime}$ has multi-peaks and appears less converged. The locations of the peak $u^{\prime}$ and $v^{\prime}$ values are now at $\eta>0$, consistent with the result $\frac{d y_{0.3}}{d x}>0$ shown in Table 2. At some stations, the shear stress may be come negative $\left(\overline{u^{\prime} v^{\prime}}>0\right)$, suggesting again the type of behavior observed in region II of a forced shear layer (Oster and Wygnanski 1982, Figs. 25, 26). Since negative shear stress is not seen in Figure 14, nonuniform density may also have some effect. The shear stress distributions at the last three stations are multipeaked and far from reaching an asymptotic state. Unlike the shear stress, the fluctuating density profiles are seen to evolve rather quickly to an asymptotic distri- 
bution, with the final, slowest adjustment occurring only near the edges of the shear layer.

The peak values of $u^{\prime}, v^{\prime}, \rho^{\prime}$ and shear stress for Cases I, III and IV are plotted as functions of $\frac{\pi}{11}$ in Figure 16. They all show a rapid initial growth at small values of $\boldsymbol{x}$. For $u^{\prime}, v^{\prime}$ and $\rho^{\prime}$, this is followed by a more gradual climb to the asymptotic values. There are oscillations in all of the results, and they are more pronounced in the peak shear stress values than in others. If randomness in amplitudes, frequencies or relative phase angles are introduced in the perturbations at the upstream boundary, vortex pairing locations will be less deterministic and these oscillations in peak values may be reduced. It may also be that, even though the present mesh distribution is adequate to capture the asymptotic distribution of the mean velocity and density, longer fine-zone computational distance in the flow direction is needed to study the asymptotic development of the shear stress distribution, especially for the higher shear variable density case.

For constant-density flows, Oster and Wygnanski (1982) found experimentally that the peak value of $u^{\prime}$ (normalized by the velocity difference across the shear layer as was done here) in the asymplotic region was approximately constant for velocity ratio $r\left(=\frac{U_{2}}{U_{2}}\right)$ between 0.3 and 0.6. As shown in Figure 16, this weak dependence of the asymptotic peak value on the velocity ratio is predicted here not only for $u^{\prime}$, but also for $v^{\prime}$. In addition, present calculations show that the peak values of $u^{\prime}$ and $v^{\prime}$ are also insensitive to density ratio; no measurements are available for comparison. The oscillatory behavior of the peak shear stress, especially for higher shear cases, precludes any definitive conclusion.

The fluctuating velocity profiles at selected stations for Case III are compared in Figure 17 with the measurements of Oster and Wygnanski(1982) and the calculations of Inoue and Leonard (1987). The present $u^{\prime}$ and shear stress results are reasonably close to the data, but the peak value of $v^{\prime}$ is about twice the measured value. It is interesting to note that, in a singlestream shear layer $(r=0)$ for initially laminar condition, Bradshaw (1966) reported a peak $v^{\prime}$ value almost twice its fully-developed asymptotic value. This overshoot was seen to occur in the early region where the flow is presumably more two-dimensional. This observation, together with the close agreement shown in Figure 17 between the present calculations and the two-dimensional results of Inoue and Leonard (1987) based on an entirely different numerical method with different initial and boundary conditions, suggests that the large difference between the predicted and measured $v^{\prime}$ profiles may be the result of two-dimensional flow approximation used in numerical simulations. Similar conclusions based on forced shear layer studies have been previously reached by Inoue and Leonard (1987) and by Ghoniem and $\mathrm{Ng}_{\mathrm{g}}$ (1987).

No measurements on the fluctuating velocity profiles for variable density cases are available for comparison. Konrad (1977) has, however, measured the density fluctuations for Case I; his data are seen to be in strong agreement with our calculations in Figure 18.

\section{CONCLUSIONS}

Numerical simulations on the dynamics of twodimensional shear layers have been carried out using an explicit second-order Godunov scheme. Quantitative results have been obtained by statistically averaging the numerical solutions over long time intervals and compared with available experimental data and theoretical calculations. Consistent with the (constantdensity) solutions of Grinstein et al. (1986) and the (variable-density) experiments of Brown and Roshko (1974), the present study shows that more high-speed fluid particles are entrained into the shear layer than low-speed fluid particles, and reducing the density of the high-speed fluid results in higher spreading rate. In addition, the slope of the momentum thickness is not indicative of the extent of mixing in a variable-density shear layer.

Furthermore, this study shows that the mean velocity and density profiles do approach an asymptotic, similar shape, and the developmental length is increased when the density of the high-speed fluid is reduced. Time-averaged fluctuating fow profiles approach their asymptotic states much slower than the mean profiles. Consistent with the experimental finding of Oster and Wygnanski (1982), the peak value of $\boldsymbol{u}^{\prime}$ in the asymptotic region is insensitive to the velocity ratio. In addition, present calculations show that this is also true for $v^{\prime}$, and the peak values of $u^{\prime}$ and $v^{\prime}$ are also insensitive to the density ratio.

In conclusion, this study suggests that the mean-flow evolution of shear layers is dominated by inviscid effects. Accurate predictions of mean velocity, mean density, r.m.s. $u^{\prime}$, shear stress and fluctuating density profiles can be made by a two-dimensional flow simulation. To improve the accuracy of r.m.s. $v^{\prime}$ profiles, however, a three-dimensional calculation is required. 


\section{LIST OF REFERENCES}

Ashurst, W.T., Turbulent Shear Flows I (1979) ed. by F. Durst, et al., Springer-Verlag, Berlin, pp. $402-413$.

Bradshaw, P. (1966) The Effect of Initial Conditions on the Development of a Free Shear Layer, J. Fluid Mech., 26, pp. 225-236.

Brown, G.L. and Roshko, A. (1974) On Density Effects and Large Structure in Turbulent Mixing Layers, J. Fluid Mech, 64, pp. 775-816.

Chien, K.-Y., Ferguson, R.E., Collins, J.P., Glaz, H.M., Kuhl, A.L. (1987) A Study of Mixing in Forced Shear Layers with an Euler Code, AIAA Paper 87-1318.

Chien, K.-Y., Ferguson, R.E., Kuhl, A.L., Glaz, H.M. and Colella, P. (1988) Inviscid Simulations of Turbulent Shear Layers-Mean Flow Analysis, International Workshop on the Physics of Compressible Turbulent Mixing, Princeton University, Princeton, N.J.

Chien, K.-Y., Ferguson, R.E., Kuhl, A.L., Glaz, H.M., and Colella, P. (1989) Inviscid Simulations of Turbulent Shear Layers-Fluctuating Flow Pro files, Seventh Symposium on Turbulent Shear Flows, Stanford University, Stanford, CA, 1, pp. 4.3.1-4.3.5.

Chorin, A.J. (1973) Numerical Studies of Slightly Viscous Flow, J. Fluid Mech. 57, pp. 785-796.

Chorin, A.J. (1986) Vortex Methods for the Study of Turbulent Combustion at a Low Mach Number, Tenth Int. Collog. on the Dynamics of Explosions and Reactive Systems, Prog. in Astronautics and Aeronautics 105, pp. 14-21.

Colella, P. and Glaz, H.M. (1985) Efficient Solution Algorithms for the Riemann Problem for Real Gases, J. Comp. Phys. 59(2), pp. 264-289.

Colella, P. Ferguson, R.E., Glaz, H.M., Kuhl, A.L. (1985) Mach Reflection from an HE-Driven Blast Wave, Dynamics of Explosions, Prog. Astro. and Aero. 106; Bowen, Leyer and Soloukhin, editors, pp. $388-421$.
Corcos, G.M. and Sherman, F.S. (1984) The Mixing Layer: Deterministic Models of a Turbulent Flow. Part 1. Introduction and the Two-Dimensional Flow, J. Fluid Mech. 139, pp. 29-65.

Davis, R.W. and Moore, E.F. (1985) A Numerical Study of Vortex Merging in Mixing Layers, Physics of Fluids 28, pp. 1626-1635.

Ghoniem, A.F., Chorin, A.J., Oppenheim, A.K. (1982) Numerical Modeling of Turbulent Flow in a Combustion Tunnel, Philos. Trans. R. Soc. London, Ser. A 904, pp. 303-325.

Ghoniem, A.F., and Ng, K.K. (1987) Numerical study of the Dynam-ics of a Forced Shear Layer, Physics of Fluids, 30, pp. 706-721.

Glaz, H.M., Colella, P., Glass, I.I. and Deschambault, R.L. (1985) A Numerical Study of Oblique ShockWave Reflections with Experimental Comparsons, Proc. Royal Soc. London, Ser. A. 398, pp. 117-140.

Glowacki, W.J., Kuhl, A.L., Glaz, H.M. and Ferguson, R.E. (1986) Shock Waves and Shock Tubes, ed. by D. Bershader and R. Hanson, Stanford University Press, Stanford, CA, pp. 187-194.

Grinstein, F.F., Oran, E.S., Boris, J.P. (1986) Numerical Simulations of Asymmetric Mixing in Planar Shear Layers, J. Fluid Mech. 165, pp. 201-220.

Ho, C.M. and Huang, L.S. (1982) Subharmonics and Vortex Merging in Mixing Layers, J. Fluid Meck. 119 , pp. $443-473$.

Inoue, O. (1985) Vortex Simulation of a Turbulent Mixing Layer, AIAA Journal 23, pp. 367-373.

Inoue, O. and Leonard, A. (1987) Vortex Simulation of Forced/Unforced Mixing Layers, AlAA Paper 87-0288; also AlAA Journal 25, pp. 1417-1418.

Konrad, J.H. (1977) An Experimental Investigation of Miring in Two-Dimensional Turbulent Shear Flows with Applications to Diffusion-Limited Chemical Reactions, Ph.D. Thesis, California Institute of Technology, Pasadena, California. 
iuhl, A.L., Colella, P., Berger, M., Glaz, H.M., Ferguon, R.E., (1987) Unstable Wall Jet Evolution for - Double-Mach Stem Flow, Eleventh Int. Colloq. on the Dynamics of Explosions and Reactive Systems.

iuhl, A.L., Chien, K.Y., Ferguson, R.E., Glaz, H.M. and Colella, P. (1990) Inviscid Dynamics of Unstable Shear Layers, DNA-TR-89-6, Defense Nuclear Agency, Alexandria, VA.

conard, A. (1980) Vortex Methods for Flow Simulation, J. Comp. Phys. 37, pp. 289-335.

iepmann, H.W. and Laufer, J. (1947) Investigation of Free Turbulent Mixing, NACA Tech. Note No. 1257.

IcInville, R.M., Gatski, T.B., Hassan, H.A. (1985) Analysis of Large Vortical Structures in Shear Layers, AIAA Joumal 23, pp. 1165-1171.

lichel, F. (1932) Larm und Resonanzschwingungen im Krafiwe rksbetriels (Noise and Resonant Vibrations in Power Plants). Berlin VDI-Verlag.

lonkewitz, P.A. and Huerre, P. (1982) Influence of the Velocity Ratio on the Spatial Instability of Mixing Layers. Phys. of Fluids 25, pp. 1137-1143.

Ister, D. and Wygnanski, 1. (1982) The Forced Mixing Layer Between Parallel Streams, J. Fluid Mech. 123, pp. 91-130.

Liley, J.J. and Metcalfe, R.W. (1980) Direct Numerical Simulation of a Perturbed Turbulent Mixing Layer, AIAA Paper 80-0274.

Loshko, A. (1981) The Plane Mixing Layer: Flow Visualization Results and Three Dimensional Effects, Lecture Notes in Physics, 136 The Role of Coherent Structures in Modelling Turbulence and Miring, ed. by J. Jimenez, Springer-Verlag, pp. 208-217.

'inant, C.D. and Browand, F.K. (1974) Vortex Pairing, the Mechanism of Turbulent Mixing Layer Growth at Moderate Reynolds Number, J. Fluid Mech., 63, pp. 237-255.

Voodward, P.R. and Colella, P. (1984) Numerical Simulation of Two-Dimensional Fluid Fiow with Strong Shocks J. Comp. Phys. 54, pp. 115-173. 
(a)

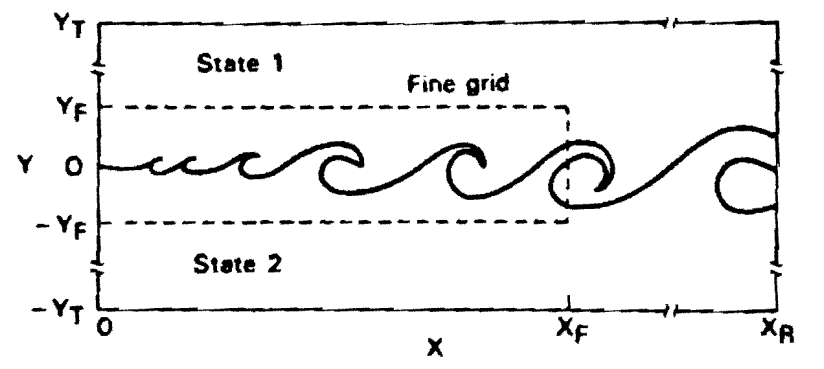

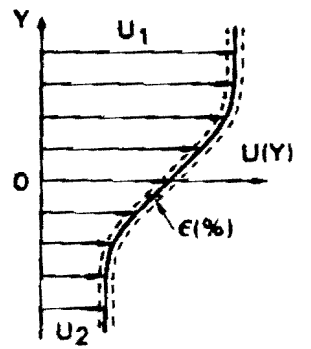

(b)

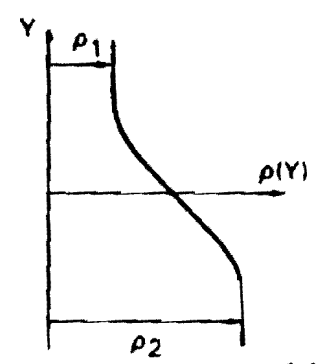

(c)
Figure 1. Schematic of the free shear layer calculations: (a) computational grid; (b) Tanh(y) streamrise velocity profile; and (c) density profile.
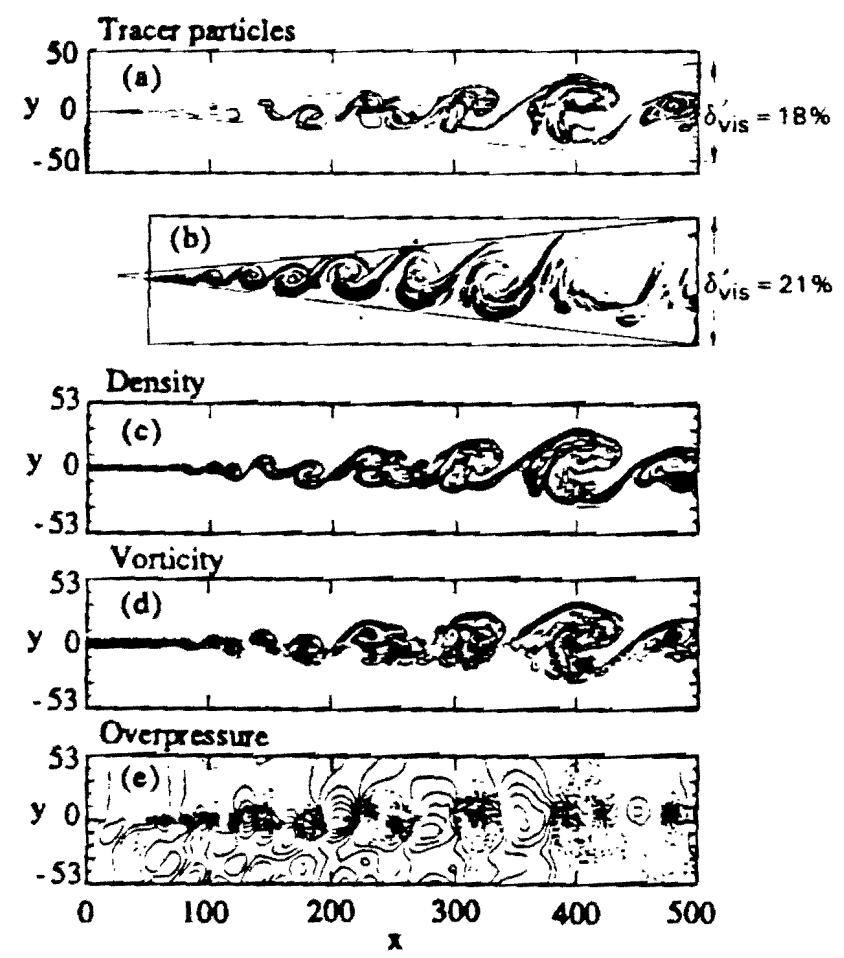

Figure 2. Flow field for Case I: (a) material interface; (b) shadowgraph from the experiment; (c) density contours; (d) vorticity contours (solid lines denote negative values); and (e) overpressure contours (solid lines denote positive values).
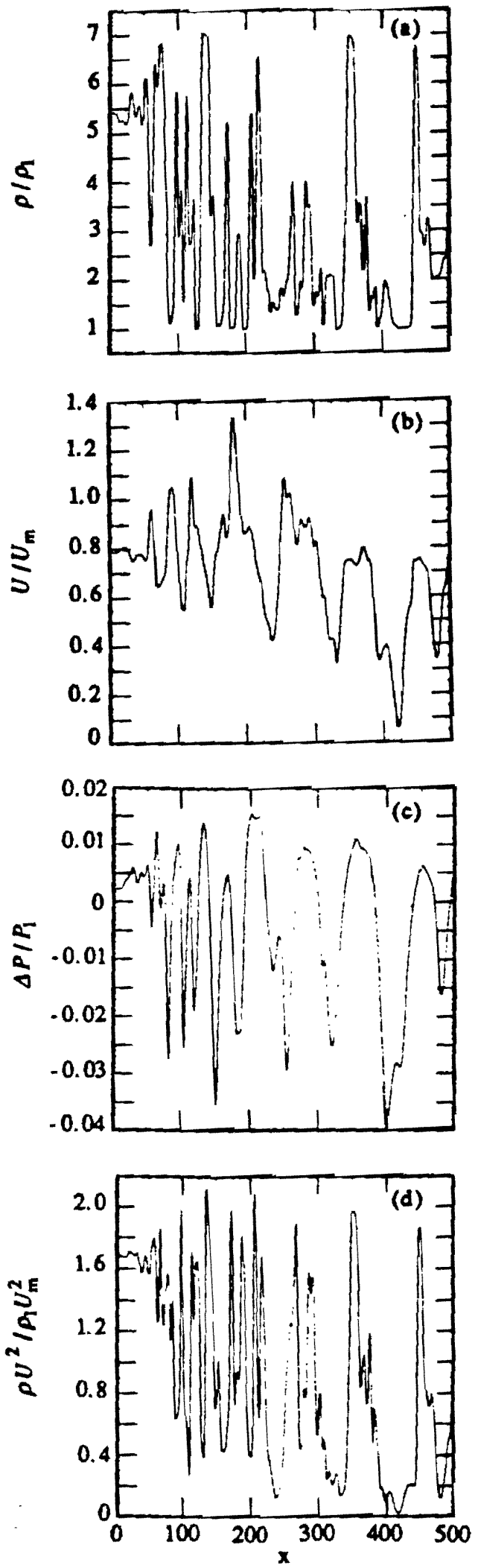

Figure 3. Flow field along the centerline (i.e., $y=$ $0.5=0.5 \Delta y$ ) corresponding to Figure 2 . 


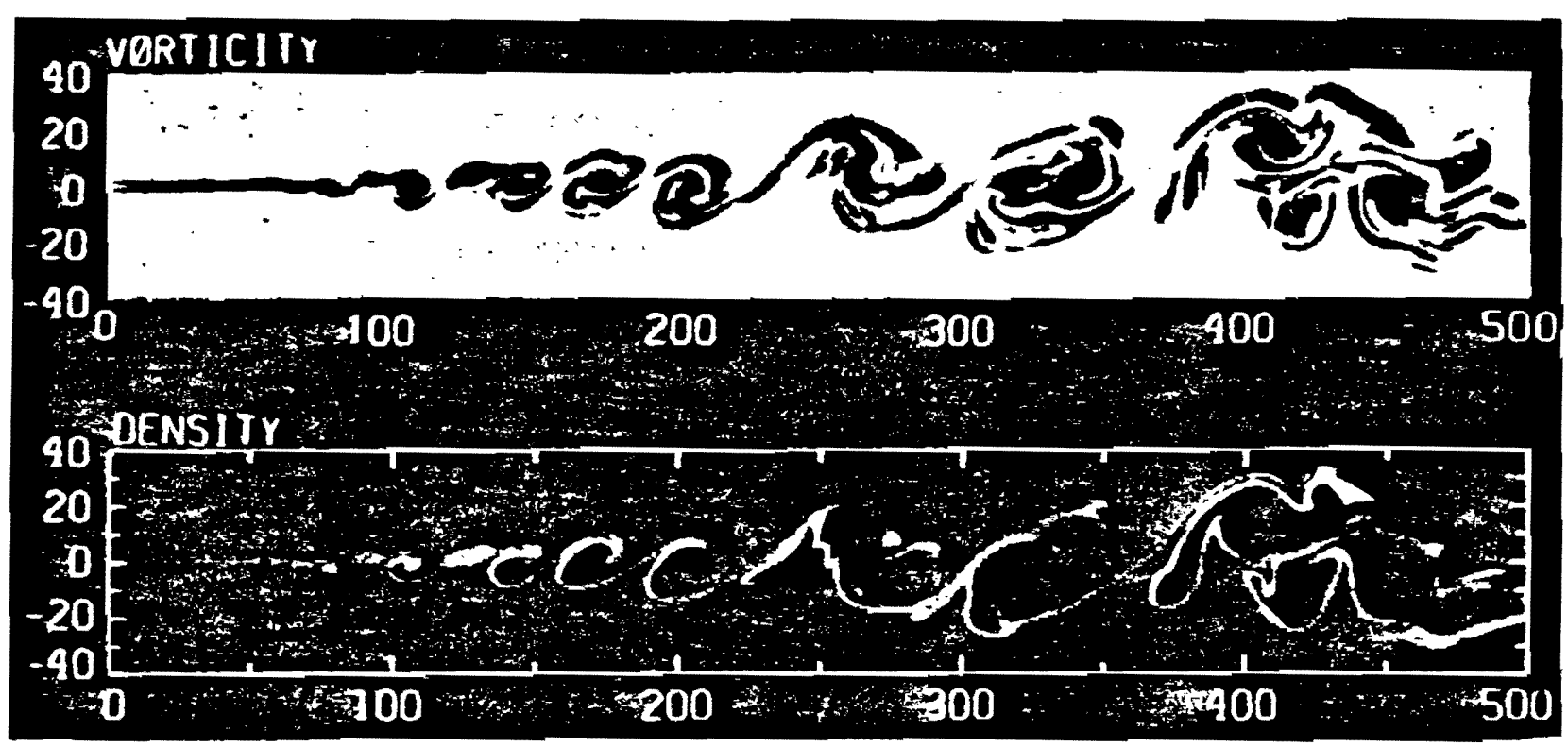

i $\quad x+4+x+4$

ten:

Figure 4. Vorticity and density color visualizations for Case I.
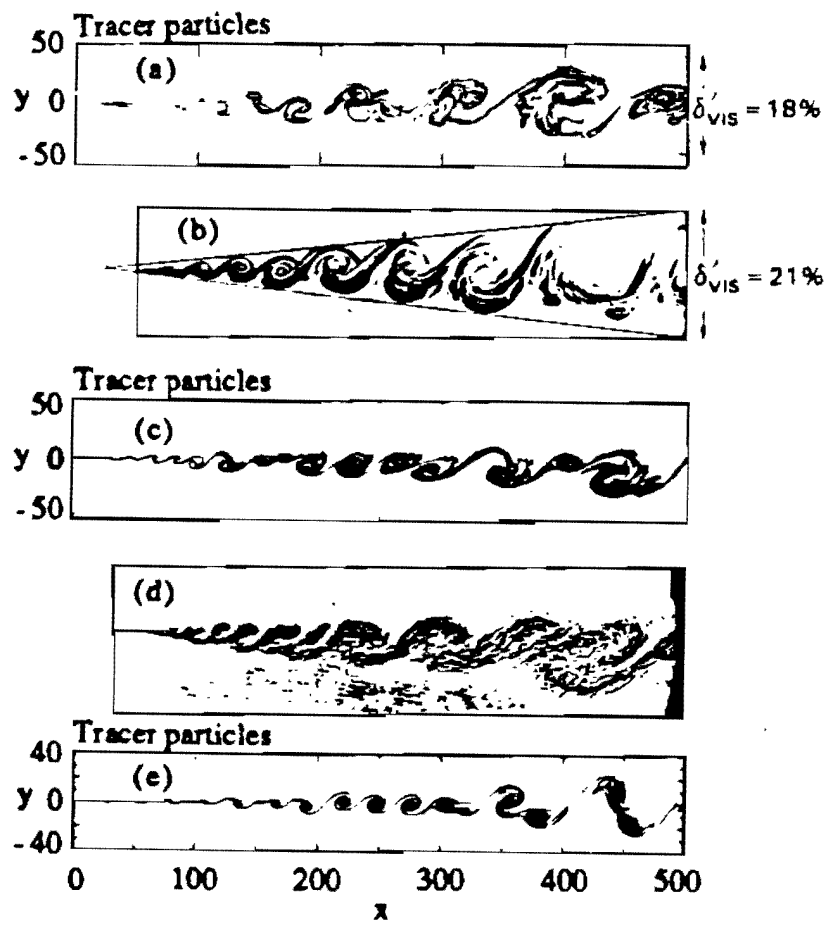

Figure 5. Material interface plots comparing the spreading shear layer cases: (a), (b) Case I, (c), (d) Case II, (e) Case III. Figures (b), (d) are photographs of the experimental record (Brown and Roshko 1974).

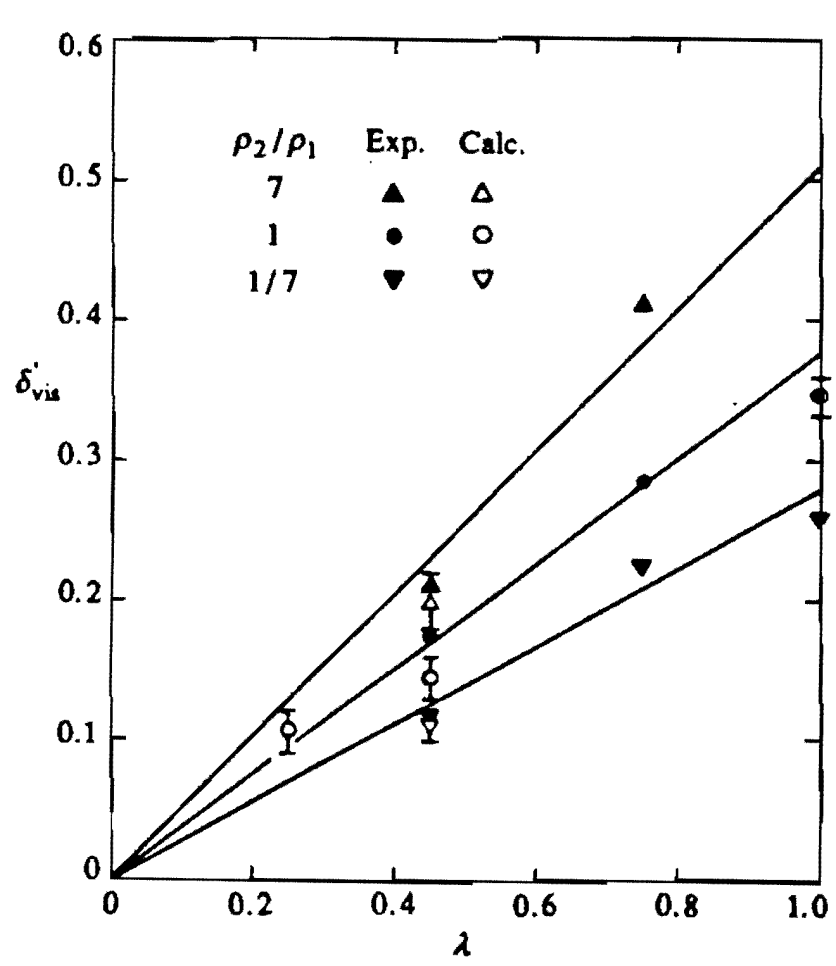

Figure 6 . Visual spreading rate, $\delta_{v i s}^{\prime}$ vs. $\lambda$, for the spreading shear layer cases. 

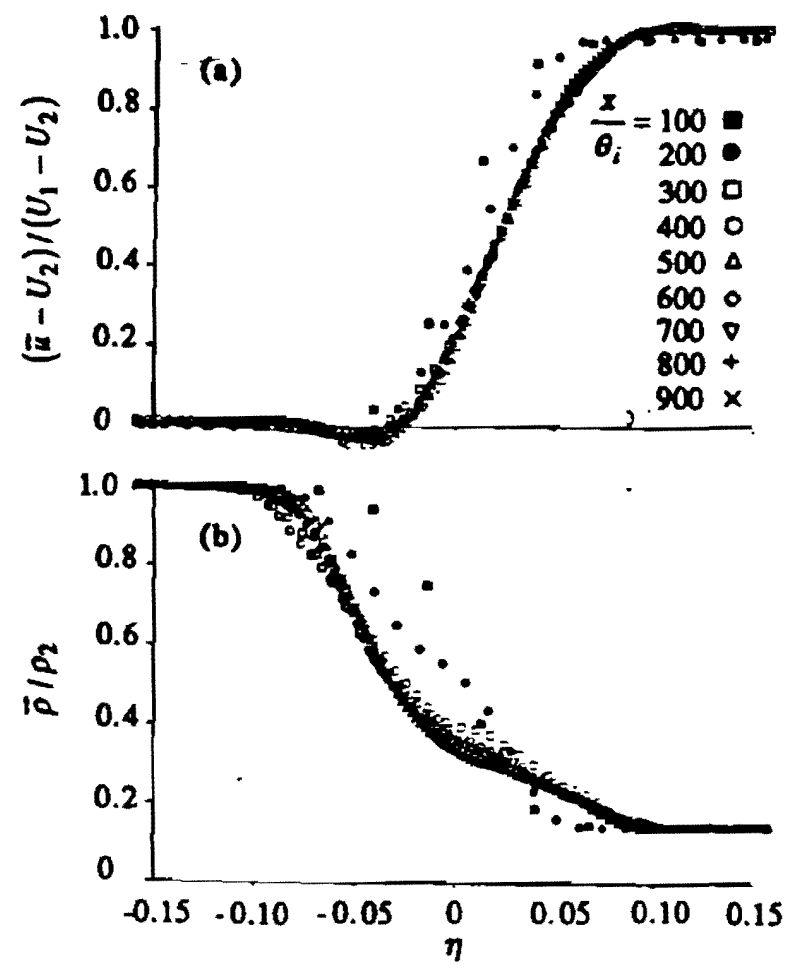

Figure 7. Mean velocity and density profiles for Case I.

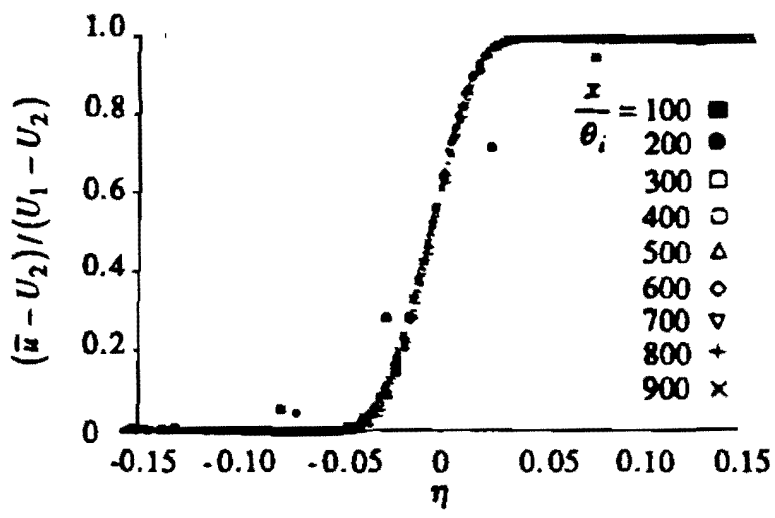

Figure 8. Mean velocity profile for Case III.

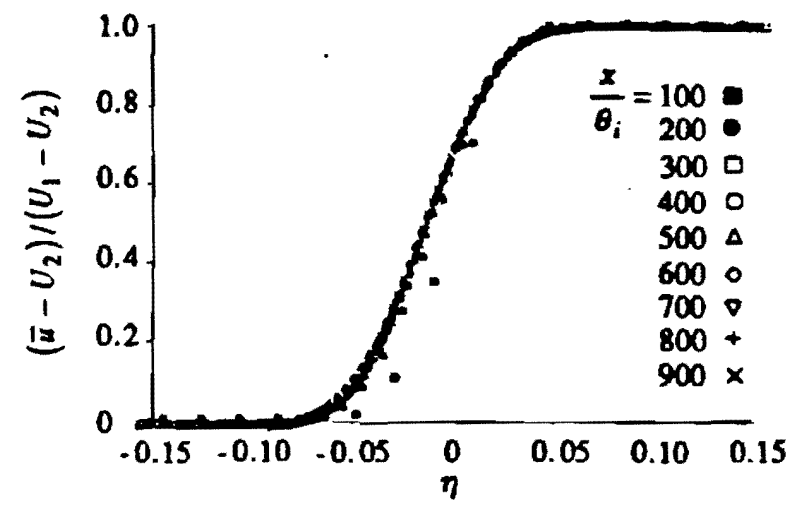

Figure 9. Mean velocity profile for Case IV.
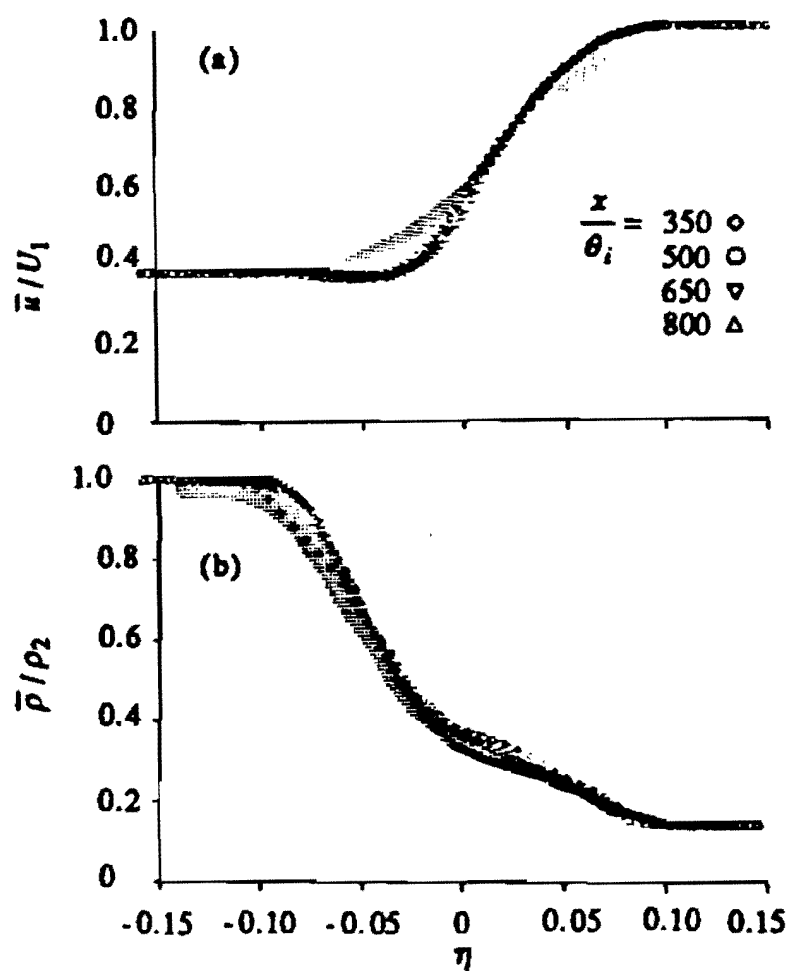

Figure 10. Mean velocity and density profiles for Case I; the shaded regions denote the experimental data band of Brown and Roshko (1974).
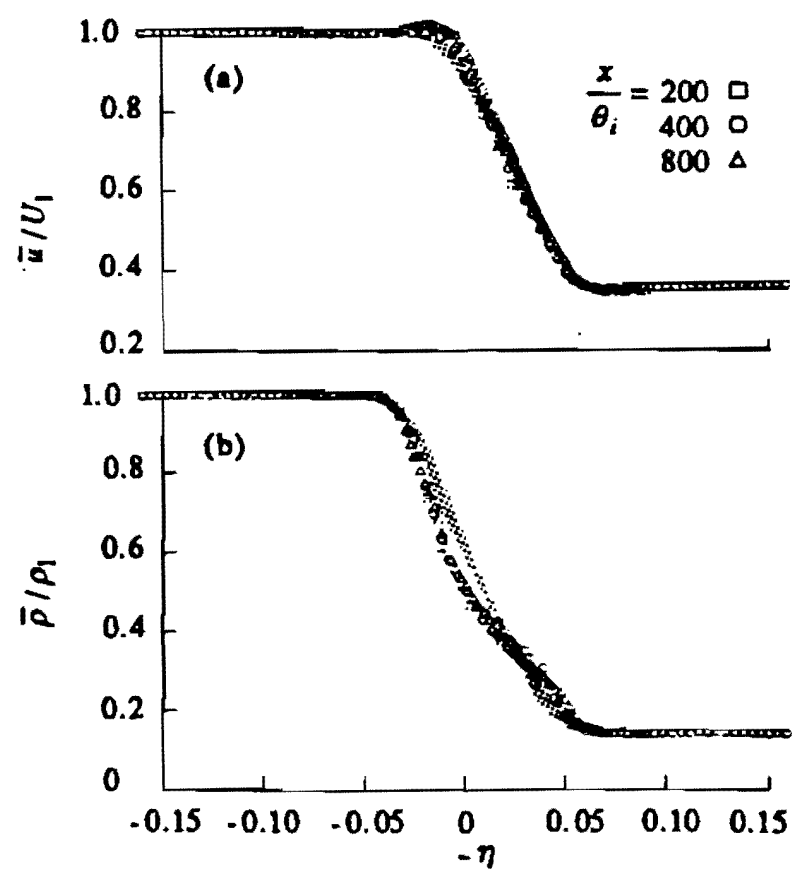

Figure 11. Mican velocity and density profiles for Case II; the shaded regions denote the experimental data band of Brown and Roshko (1974). 


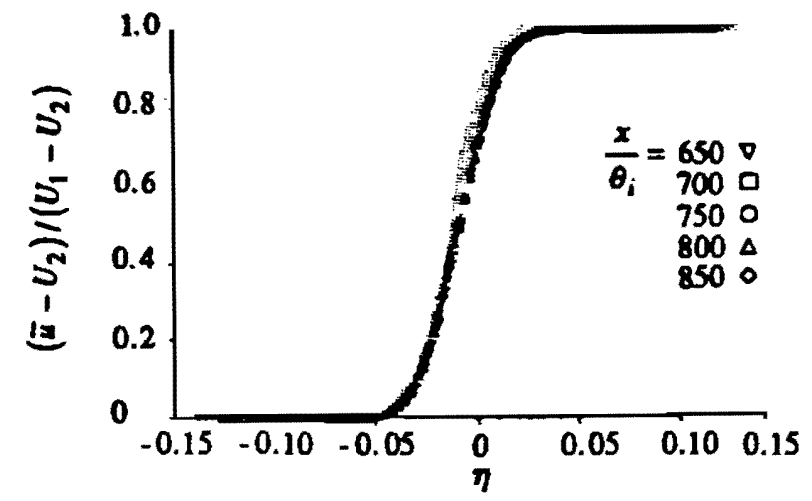

Figure 12. Mean velocity profiles for Case III; the shaded region denotes the experimental data band of Oster and Wygnanski (1982).
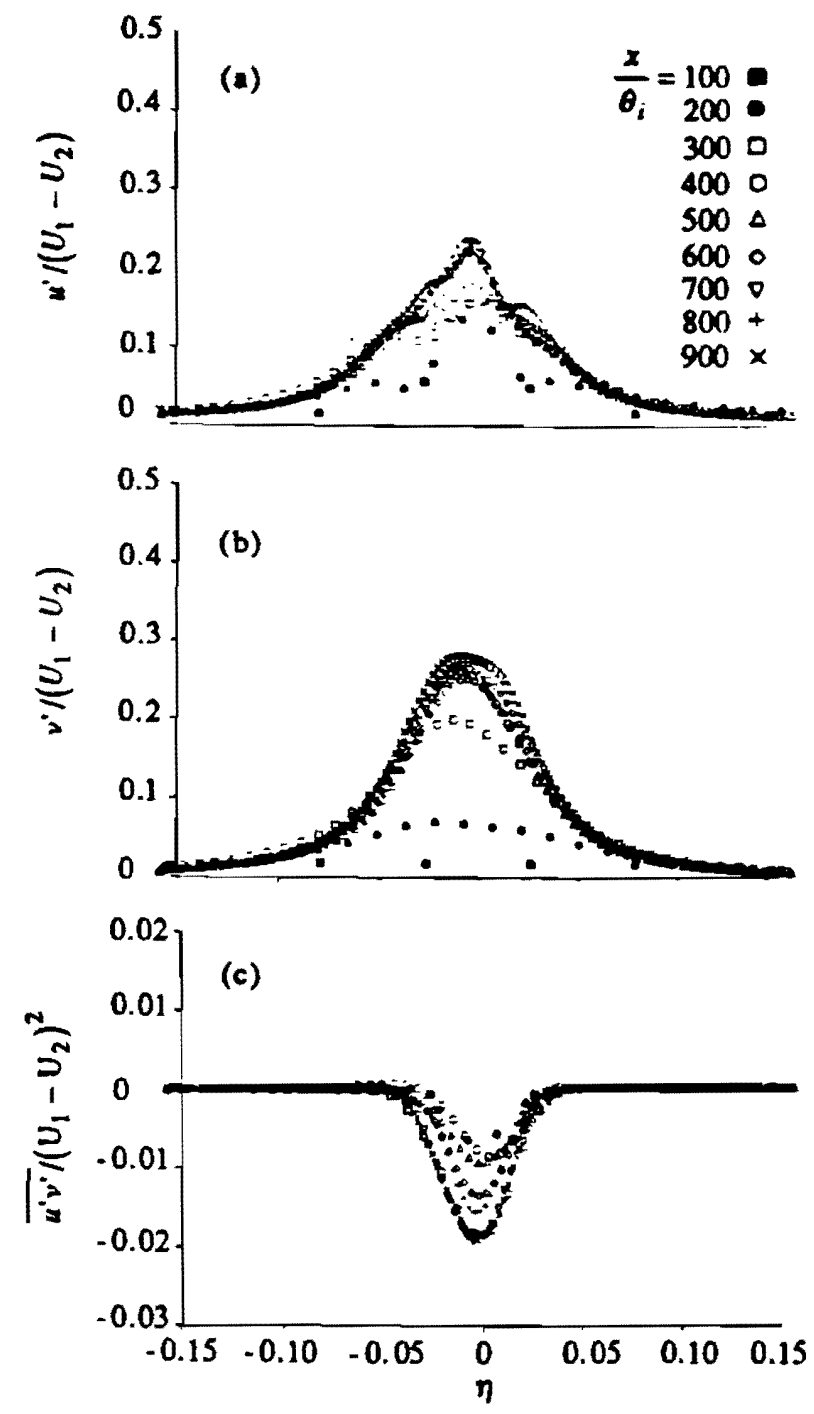

Figure 13. Time-averaged fluctuating-flox profiles for Case III: (a) streamwise velocity; (b) transverse velocity; (c) shear stress.
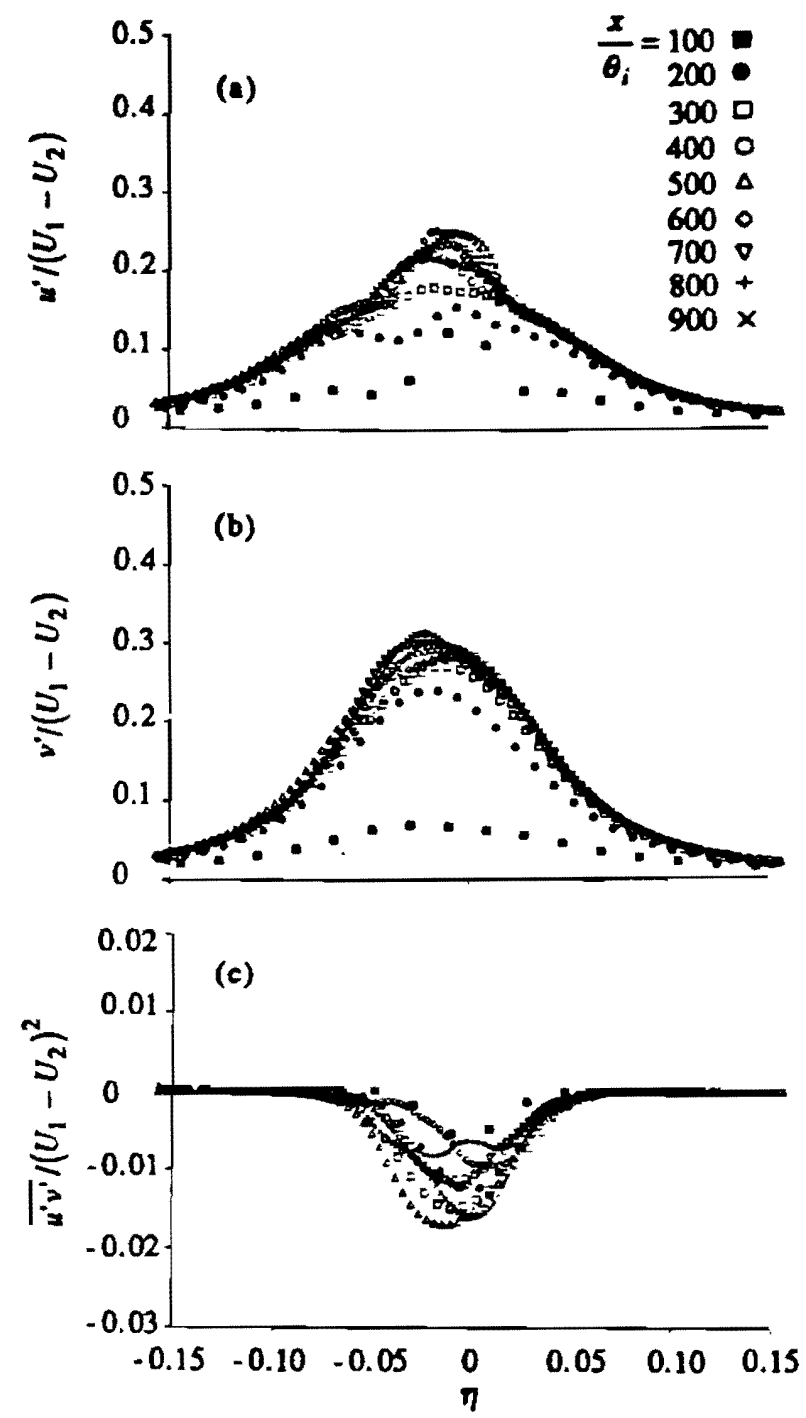

Figure 14. Time-averaged fluctuating-flow profiles for Case IV: (a) streamwise velocity; (b) transverse velocity; (c) shear stress. 

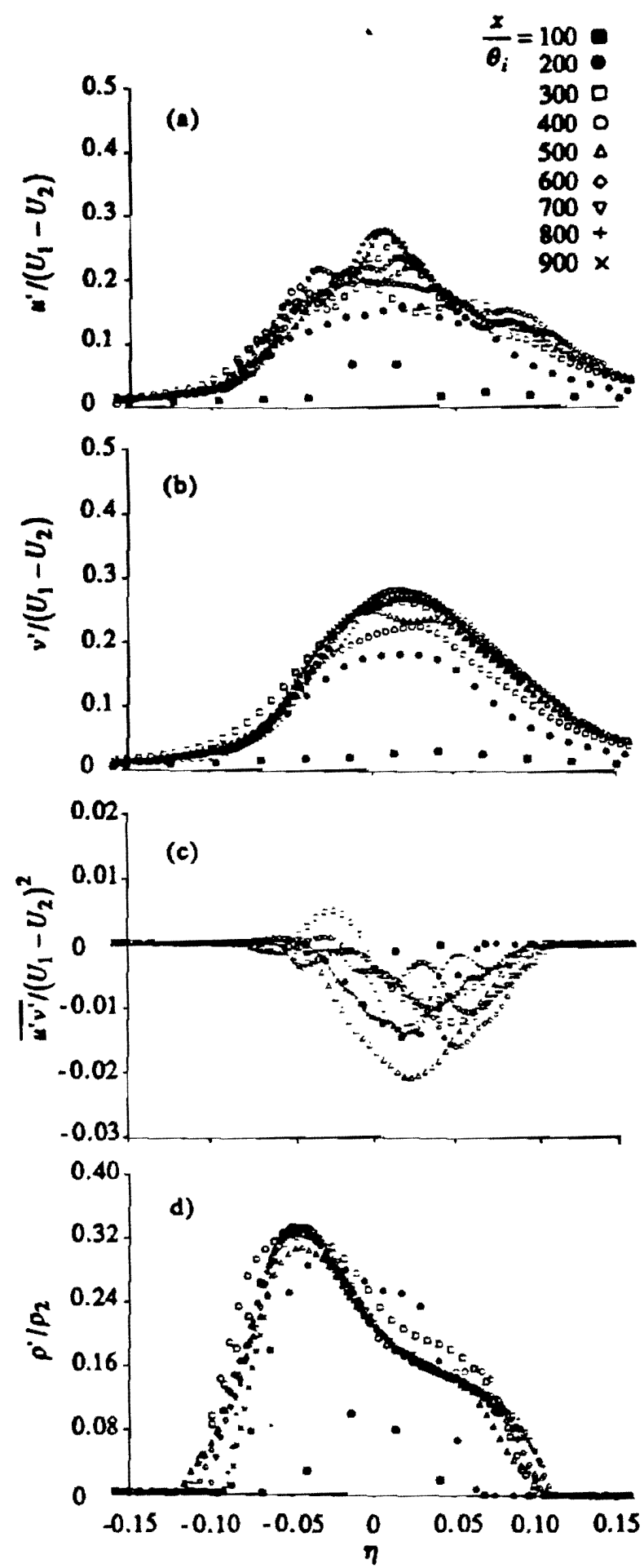

Figure 15. Time-averaged fluctuating-flow profiles for Case I: (a) streamwise velocity; (b) transverse velocity; (c) shear stress; (d) density.
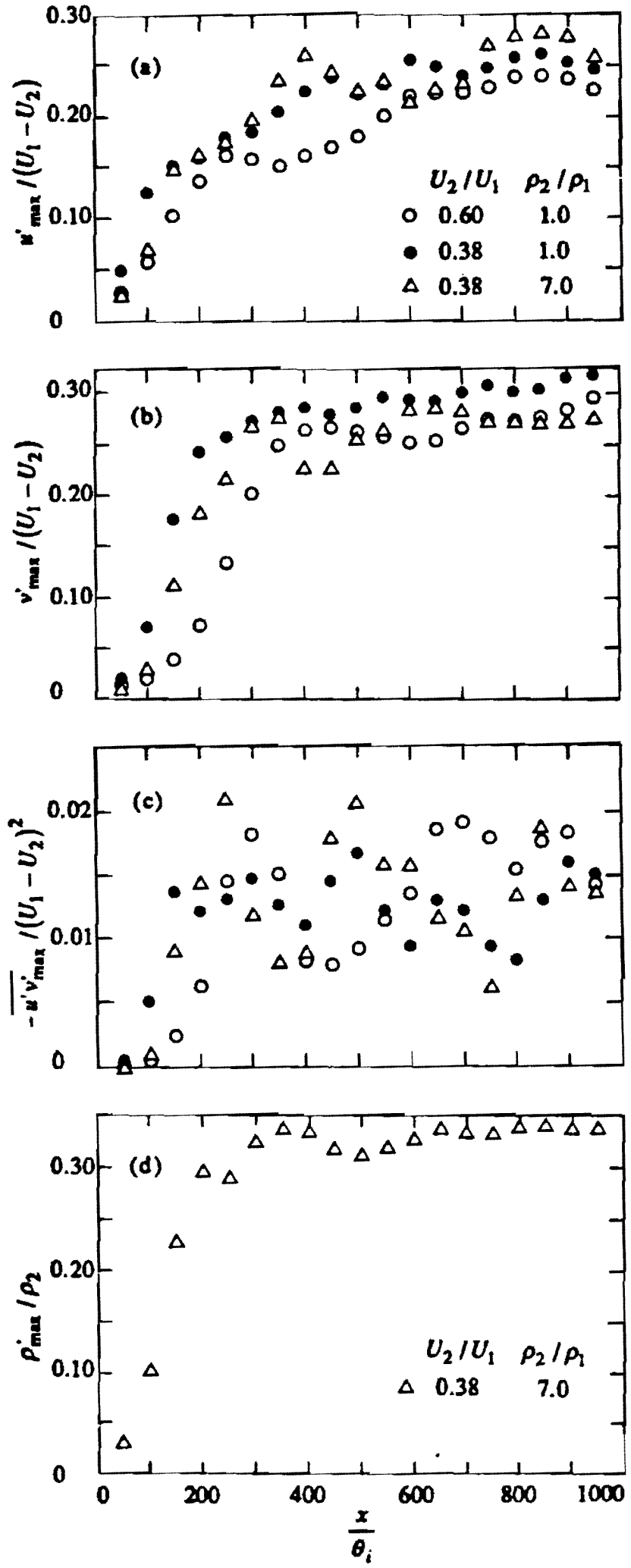

Figure 16. Peak t.m.s. values: (a) streamwise velocity; (b) transverse velocity; (c) shear stress; (d) density. 

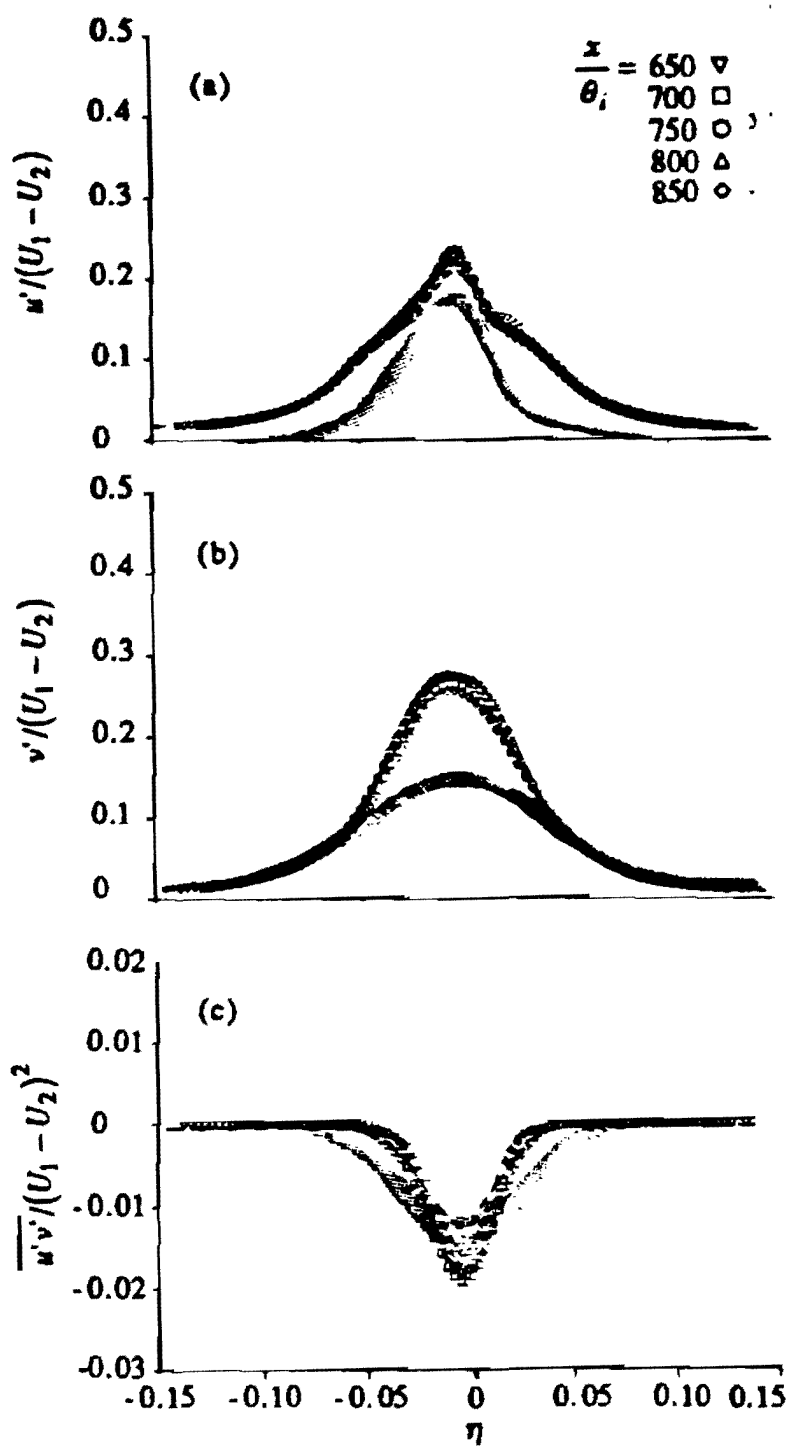

Figure 17. Time-averaged fluctuating-flow profiles for Case III: (a) streamwise velocity; (b) transverse velocity; (c) shear stress. Shaded regions denote the data of Oster and Wygnanski (1982). The dashed lines represent the profiles calculated with a $2 \mathrm{D}$ vortex dynamics code (Inove and Leonard 1987).

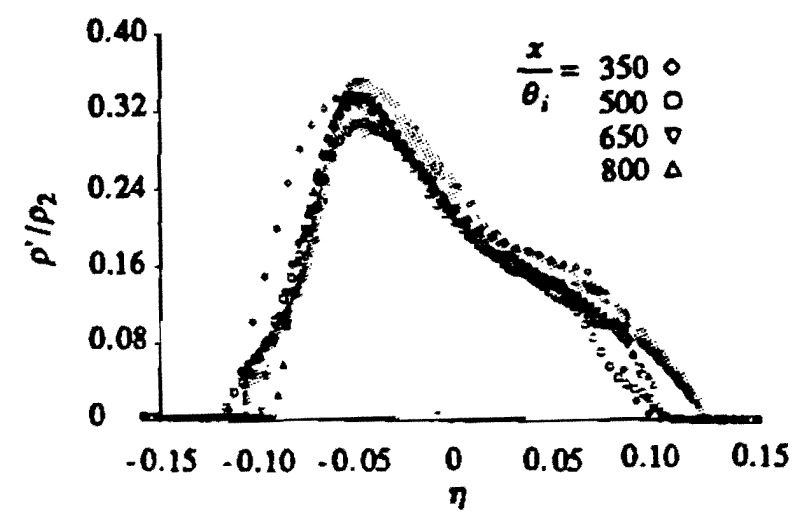

Figure 18. Time-averaged fluctuating density profiles for Case I. The shaded region denotes the experimental data band of Konrad (1977). 
.. NOTES .. 
.. NOTES .. 